\title{
POLLINATION SYSTEMS AND FLORAL TRAITS IN CERRADO WOODY SPECIES OF THE UPPER TAQUARI REGION (CENTRAL BRAZIL)
}

\author{
MARTINS, F. Q. and BATALHA, M. A. \\ Department of Botany, Federal University of São Carlos, C. P. 676, CEP 13565-905, São Carlos, SP, Brazil \\ Correspondence to: Marco Antônio Batalha, Department of Botany, Federal University of São Carlos, \\ C. P. 676, CEP 13565-905, São Carlos, SP, Brazil, e-mail: marcobat@uol.com.br \\ Received November 8, 2004 - Accepted February 16, 2005 - Distributed May 31, 2006
}

(With 1 figure)

\begin{abstract}
Plant species present flowers with varied morphological and functional features, which may be associated to pollination systems, including species pollinated by wind, beetles, moths, bees, small insects, birds, or bats. We calculated the frequencies of the pollination systems among woody species in five cerrado fragments in central-western Brazil and tested whether the pollination systems were indeed related to floral traits. We sampled 2,280 individuals, belonging to 121 species, ninety-nine of which were described in relation to all floral traits. Most species had diurnal anthesis, pale colors, and open flowers. The most frequent groups were those composed by the species pollinated by bees, small insects, and moths. A Principal Component Analysis of the species and floral traits showed that there was a grouping among species with some pollination systems, such as those pollinated mainly by beetles, moths, birds, and bats, for which inferences based on the floral traits are recommended in cerrado sites. For the species pollinated mainly by bees or small insects, inferences based on the floral traits are not recommended, due to the large dispersion of the species scores and overlapping between these two groups, which probably occurred due to the specificity absence in plant-pollinator relationships.
\end{abstract}

Keywords: cerrado, phenology, pollination, principal components analysis, savanna.

\section{RESUMO}

\section{Sistemas de polinização e caracteres florais em espécies lenhosas de cerrado na região do Alto Taquari (GO, MS e MT)}

As espécies vegetais apresentam flores com características morfológicas e funcionais diversificadas, que podem ser associadas a sistemas de polinização, incluindo espécies polinizadas pelo vento, besouros, mariposas, abelhas, insetos pequenos, aves ou morcegos. Calculamos as frequiências dos sistemas de polinização entre as espécies lenhosas em cinco fragmentos de cerrado no Brasil central e testamos se os sistemas de polinização estavam de fato relacionados às características florais. Amostramos 2.280 indivíduos, pertencentes a 121 espécies, das quais 99 foram descritas em relação a todas as características florais. A maioria dessas espécies possuía antese diurna, cores claras e flores abertas. Os grupos mais freqüentes foram os das plantas polinizadas por abelhas, insetos pequenos e mariposas. Uma análise de ordenação das espécies e dos caracteres florais mostrou que houve agrupamentos entre as espécies com alguns sistemas de polinização, tais como as polinizadas principalmente por besouros, mariposas, aves e morcegos, para os quais inferências baseadas em características florais são recomendadas. No caso das espécies polinizadas por abelhas e insetos pequenos, inferências a partir dos caracteres florais não são recomendadas devido à grande dispersão dos escores das espécies e à sobreposição entre esses dois grupos, que ocorreram, provavelmente, devido à ausência de especificidade nas relações planta-polinizador.

Palavras-chave: análise de componentes principais, cerrado, fenologia, polinização, savana. 


\section{INTRODUCTION}

Savannas are tropical and subtropical formations where the grass layer is almost continuous, interrupted only by shrubs and trees in varying proportions, and where the main growth patterns are closely associated with alternating wet and dry seasons (Bourlière \& Hadley, 1983). The Cerrado domain formerly occupied 2 million $\mathrm{km}^{2}$ of the Brazilian territory (Ratter et al., 1997), especially in the Central Plateau. As its name implies, in the Cerrado domain, the cerrado vegetation prevails. The cerrado vegetation is not uniform in physiognomy (Coutinho, 1990), ranging from grassland to tall woodland, but with most of its physiognomies within the range defined as tropical savanna (Bourlière \& Hadley, 1983). The cerrado vascular flora has an herbaceous and a woody component, which are antagonistic because both are heliophylous (Coutinho, 1990). Scholes \& Archer (1997) postulated that the climatic seasonal pattern of tropical savannas, with alternating warm dry and hot wet seasons, provides a potential axis of niche separation by phenology for the herbaceous and woody components.

Plant reproductive processes are determinants of the composition and structure of communities (Bawa, 1990; Oliveira \& Gibbs, 2000). Among such processes, the plant-pollinator interactions form a dynamic, yet somewhat cohesive, ecological subunit of a community (Moldenke \& Lincoln, 1979). Pollination biology at community level in the Neotropics has been studied in forest areas (Bawa et al., 1985; Kress \& Beach, 1994) and also in the cerrado vegetation (Silberbauer-Gottsberger \& Gottsberger, 1988; Barbosa, 1997; Oliveira \& Gibbs, 2000). Pollination is an important ecological process for vegetation communities since it can directly affect the reproductive success of plants, being able to cause loss of species if affected (Wunderlee, 1997). Pollination usually involves abiotic (water or wind) or biotic (animal) vectors, including from non-specialist insects to animals strictly dependent on flowers for their survival, such as bees, birds, and bats (Proctor et al., 1996; Machado \& Oliveira, 2000).

Plant species have flowers with many morphologic and functional features, which can be associated to pollination syndromes (Faegri \& Pijl, 1979; Borges, 2000). These syndromes are composed of a set of attributes (e.g., color, odor, shape, reward, and anthesis), which are common or specific to plant species adapted to a certain type of pollinator (Faegri \& Pijl, 1979; Waser et al., 1996). Pollination syndromes take into account these set of floral characters, which would allow determining the likeliest group of pollinators in certain species (Bawa et al., 1985). However, the concept of pollination syndromes must be applied with caution (Herrera, 1996), since it presupposes pollinator-plant specialization, which, in general, is rare (Waser et al., 1996). Pollination systems with a high level of specialization are exceptions; they often vary from less specialized to more generalized systems (Waser et al., 1996). Thus, Oliveira \& Gibbs (2000) grouped species according to pollination systems or functional guilds. In this sense, pollination service from different types of animals may be seen as a resource that unifies different pollination systems (Oliveira \& Gibbs, 2000).

It is important to consider the attractive and floral resources displayed by species to their visitors, since these aspects ensure the presence and the fidelity of pollinators to the flowers (Borges, 2000). Pollinators are able to distinguish floral differences and have preferences for some colors, forms, sizes, and odors (Levin \& Anderson, 1970). Cerrado species ensure the attraction and permanence of pollinators in their environment, offering resources in morphologically different flowers (Borges, 2000). This attraction is reinforced in some species by the emission of odors, and in other ones, by morphologic characteristics, so that these features act as guides of resources (Borges, 2000). Bees are considered better-adapted animals to pollination (Faegri \& Pijl, 1979) and are the main pollinators of tropical areas (Bawa, 1990; Ramirez \& Brito, 1992).

In the cerrado vegetation, bees are also the main pollinators (Silberbauer-Gottsberger \& Gottsberger, 1988; Barbosa, 1997; Borges, 2000; Oliveira \& Gibbs, 2000). Nevertheless, other pollinators are also important for the cerrado flora, since most species rely on a wide spectrum of pollinators, defined more by their size and foraging requirements than by specific interaction (Oliveira \& Gibbs, 2002). Many species have small, apparently generalist flowers, pollinated by a range of insects of different groups, such as 
flies, bees, and wasps (Oliveira \& Gibbs, 2002). As in other tropical communities, plant-pollinator relationships in cerrado seem to involve guilds of pollinators associated with a given plant or group of plants (Bawa, 1990; Oliveira \& Gibbs, 2000). Studies on the reproductive biology of cerrado plants have shown a great diversity of pollination systems, which are similar to those found in Neotropical forests (Oliveira \& Gibbs, 2002), but is it possible to infer the main pollination system of cerrado woody species based on floral traits? We studied the woody component of some cerrado fragments in central Brazil, attempting to answer the following questions: What are the main pollination systems among these species? What are their floral traits? Do species with a given pollination system form distinct groups when all their floral traits are taken into account? Which floral traits are related to a given pollination system?

\section{MATERIAL AND METHODS}

We carried out this study in 2003 in Alcinópolis (Mato Grosso do Sul State), Alto Araguaia and Alto Taquari (Mato Grosso State), and Mineiros and Santa Rita do Araguaia (Goiás State), central-western Brazil, in the southwestern extremity of the Brazilian Central Plateau. The regional climate is Aw (Köppen, 1948), humid tropical with a wet summer and dry winter. Annual rainfall varies from 1,200 to $2,000 \mathrm{~mm}$, concentrated from October to March, and the mean annual temperature is around $24.6{ }^{\circ} \mathrm{C}$ (Ramos-Neto \& Pivello, 2000). This region was originally covered mainly by cerrado vegetation, from open (campo limpo, a grassland savanna) to closed (cerradão, a tall woodland) physiognomies, following Coutinho's (1990) classification.

Based on satellite images, we randomly picked up five fragments covered mainly by cerrado sensu stricto (a woodland). The first fragment (approximately, 22K0283659S and 8036276W UTM), with 8,278 ha and a perimeter of $124.6 \mathrm{~km}$, was composed by cerrado $(90.81 \%)$, seasonal and gallery forest $(0.16 \%)$, and other vegetation types $(9.03 \%)$. The second - and the smallest - fragment (approximately, 22K0279934S and $8043454 \mathrm{~W}$ UTM), with 1,678 ha and a perimeter of $35.7 \mathrm{~km}$, was composed by cerrado $(85.62 \%)$ and other vegetation types $(14.38 \%)$. The third - and the largest - fragment (approximately, 22K0267453S and $8056613 \mathrm{~W}$ UTM), with 41,452 ha and a perimeter of $813.6 \mathrm{~km}$, was composed by cerrado $(92.11 \%)$, seasonal and gallery forest $(0.29 \%)$, and other vegetation types $(7.60 \%)$. The fourth fragment (approximately, 22K0240604S and $8039048 \mathrm{~W}$ UTM), with 6,666 ha and a perimeter of $206.0 \mathrm{~km}$, was composed by cerrado (58.67\%), seasonal and gallery forest $(32.34 \%)$, and other vegetation types $(8.99 \%)$. The fifth fragment (approximately, 22K0215774S and 7990493W UTM), with 12,459 ha and a perimeter of $386.0 \mathrm{~km}$, was composed by cerrado (40.75\%), seasonal and gallery forests $(26.54 \%)$, and other vegetation types $(32.71 \%)$. All fragments were located inside private properties and surrounded by agriculture and pasture.

We randomly placed 38 transects, perpendicular to the edge, in the cerrado sensu stricto physiognomy of the five fragments: eight in the first and fourth, four in the second, twelve in the third; and six in the fifth. In each transect, we placed 15 sampling points, $10 \mathrm{~m}$ apart one from the other, starting at $10 \mathrm{~m}$ from the fragment edge. We used the point-quarter method (Mueller-Dombois \& Ellenberg, 1974) to sample the woody component. At each point, we sampled four woody plants with a stem diameter at soil level equal to or larger than $3 \mathrm{~cm}$ (SMA, 1997). Thus, in the 38 transects, we placed 570 points and sampled 2,280 individuals. We collected the sampled individuals and identified them by comparison with lodged material at the São Paulo Botanical Institute (SP), by comparison with the Emas National Park reference collection (Batalha \& Martins, 2002), or by using a key based on vegetative characters (Batalha \& Mantovani, 1999).

We classified species into families following Judd et al. (1999) and determined their floral traits based on the literature (e.g., Barbosa, 1997; Borges, 2000; Oliveira \& Gibbs, 2000; Wanderley et al., 2002; Wanderley et al., 2003). We classified flowers according to their morphologic features (color and shape), functional features (time of anthesis, presence of odor, and presence of resource guides), and reward offered to pollinators (nectar and pollen). We considered the classes "open", "closed" and "tubular" for flower shape and "white", "cream", "green”, "lilac", "red", “orange" and "yellow" for flower color. 
We classified those species for which we determined all floral traits into pollination systems based on the literature (e.g., Silberbauer-Gottsberger \& Gottsberger, 1988; Barbosa, 1997; Borges, 2000; Oliveira \& Gibbs, 2000). Occasionally, when we could not find data for a given species, we classified its pollination system based on data for related species of the same genus. We recognized the following pollination systems: wind, beetles, moths, bees, small insects, hummingbirds, and bats, calculating the frequency of each system among the sampled individuals. The 'small insects' class included a varied assemblage of relatively small insects, such as small bees, beetles, flies, wasps, and butterflies (Bawa et al., 1985).

We constructed a matrix of the floral traits in relation to the species, excluding those species for which we did not find information for all floral traits. For the two nominal variables - flower shape and flower color, we created dummy variables, which had value 1 when a given trait was present and value 0 when it was absent (Jongman et al., 1995); for example, the dummy variable for tubular flowers had value 1 when the flower was tubular and value 0 when the flower was closed or open. To test for relationships between pollination systems and floral traits, we used a Principal Components Analyses (PCA) (Jongman et al., 1995) with the Multivariate Statistical Package software (Kovach, 1999).

\section{RESULTS}

In the 2,280 sampled individuals, we found 121 species, and for 99 of which we obtained information for all floral traits (Table 1). These 99 species comprised 2,085 individuals and 30 families. The most representative families were Myrtaceae (18 species), Fabaceae (17), and Apocynaceae, Malpighiaceae, and Melastomataceae, each one with five species. Most species had flowers with diurnal anthesis (79\%) and pale colors (89\%), that is, white, cream, yellow, or green. In relation to the shape, $68 \%$ of the species had open flowers; $24 \%$, tubular flowers; and $8 \%$, closed flowers. Pollen was a resource present in $65 \%$ of the species; and nectar, in $54 \%$. Of the 99 species, $55.6 \%$ were mainly pollinated by bees; $20.2 \%$, by small insects; $13.1 \%$, by moths; $5.0 \%$, by bats; $3.0 \%$, by beetles; $2.0 \%$, by hummingbirds; and $1.0 \%$, by wind.
In the PCA, the eigenvalues of the first two axes were 0.73 and 0.37 , explaining $46.8 \%$ of the variation in the data. In the ordination diagram (Fig. 1), the floral traits with longer vectors were shape, reward, anthesis, and, to a lesser extent, odor. Species pollinated by moths formed a distinct group, related to nocturnal anthesis and tubular flowers. These two characters, together with the presence of odor, were also related to the species pollinated by beetles and bats. Species pollinated by birds presented positive scores in the first axis and negatives scores in the second one, being related to tubular flowers, diurnal anthesis, presence of nectar, and showy colors. The only species we found pollinated by wind presented negative scores in both axes, with green, diurnal, and open flower. Species pollinated by bees and small insects were dispersed throughout both axes, one considerably overlapping with the other.

\section{DISCUSSION}

All plant species have particular morphological and physiological features that can attract some groups of floral visitors to the detriment of others (Bosch et al., 1997). Many morphological and functional aspects of floral biology are important for the establishment of plant-animal interaction (Faegri \& Pijl, 1979; Waser, 1983). Shape and odor are floral traits that have been included in community studies, because they have important implications not only for the plant-pollinator relationship, but also for the reproductive success of the plant (Endress, 1994). Flower color was particularly good at separating bird- and hymenopteran-flowers for Pentstemon species (Wilson et al., 2004). For us, however, color had a minor importance in separating pollination systems, since the two largest pollinator classes, bees and small insects, visit flowers of several colors. The exception was white, which had a relatively long vector, and was associated with moths, beetles, and bats in the second axis. We observed that the floral traits with longer vectors and, thus, best related to the pollination systems were flower shape, reward, anthesis, and odor.

Generally, the relationships between floral traits and pollination systems we found were similar to those described in other studies (e.g., Faegri \& Pijl, 1979; Silberbauer-Gottsberger \& 
TABLE 1

Floral traits and pollination systems of woody species in cerrado fragments located in Upper Taquari region (central-western Brazil). Family names were abbreviated according to Weber (1982). Pol - pollination systems; CO - color; A - anthesis; RG - resource guide; $\mathrm{O}$ - odor; $\mathrm{N}$ - nectar; $\mathrm{P}$ - pollen; $\mathrm{S}$ - shape; ba - bats; be - bees; bt - beetles; hb - hummingbird; mo - moths; si - small insects; wi - wind; cr - cream; gr - green; li - lilac; or - orange; re - red; wh - white; ye - yellow; d - diurnal; n - nocturnal; 0 - absent; 1 - present; cl - closed; op - open; tu - tubular. Sources: 1- Barbosa (1997), 2- Barros (1992), 3- Barros (1998), 4- Barros (2001), 5 - Bawa et al. (1985), 6- Borges (2000), 7- Crestana \& Kageyama (1989), 8- Franco (1995), 9- Freitas \& Oliveira (2002), 10- Gibbs et al. (1999), 11- Goldenberg (1994), 12- Goldenberg \& Shepherd (1998), 13- Gottsberger (1994), 14- Gottsberger (1999), 15- Oliveira \& Gibbs (1994), 16- Oliveira \& Gibbs (2000), 17- Oliveira \& Paula (2001), 18- Oliveira \& Sazima (1990), 19- Oliveira et al. (1992), 20- Oliveira et al. (2004), 21- Proença \& Gibbs (1994), 22- Saraiva et al. (1988), 23- Sazima \& Sazima (1975), 24- Sigrist (2001), 25- Silberbauer-Gottsberger \& Gottsberger (1988), 26- Silberbauer-Gottsberger et al. (2003), 27- Torezan-Silingardi \& Del-Claro (1998), 28- Viana et al. (1997); *classification of the pollination systems based on the genus.

\begin{tabular}{|c|c|c|c|c|c|c|c|c|c|c|}
\hline Family & Species & Pol & CO & $\mathbf{A}$ & RG & $\mathbf{O}$ & $\mathbf{N}$ & $\mathbf{P}$ & $\mathbf{S}$ & Source \\
\hline ANA & Anacardium humile A. St-Hil. & si & wh & $\mathrm{d}$ & 0 & 1 & 1 & 0 & op & $1^{*}$ \\
\hline ANN & Annona coriacea Mart. & $\mathrm{bt}$ & ye & $\mathrm{n}$ & 0 & 1 & 0 & 1 & $\mathrm{cl}$ & 13 \\
\hline ANN & Annona crassiflora Mart. & bt & ye & $\mathrm{n}$ & 0 & 1 & 0 & 1 & $\mathrm{cl}$ & 13,16 \\
\hline ANN & Bocageopsis mattogrossensis (R.E. Fries) R.E. Fries & si & wh & $\mathrm{d}$ & 0 & 0 & 0 & 1 & op & $14^{*}, 26^{*}$ \\
\hline ANN & Duguetia furfuracea (A. St-Hil.) Benth. \& Hook.f. & $\mathrm{bt}$ & re & $\mathrm{n}$ & 0 & 1 & 0 & 1 & op & 6,13 \\
\hline API & Didymopanax macrocarpum (Cham. \& Schltdl.) Seem. & si & $\mathrm{gr}$ & $\mathrm{d}$ & 0 & 1 & 0 & 1 & op & 16 \\
\hline API & Didymopanax vinosum (Cham. \& Schltdl.) Marchal & si & $\mathrm{gr}$ & $\mathrm{d}$ & 0 & 1 & 0 & 1 & op & $16^{*}$ \\
\hline $\mathrm{APO}$ & Aspidosperma macrocarpon Mart. & mo & $\mathrm{cr}$ & $\mathrm{n}$ & 1 & 1 & 1 & 0 & tu & 16,20 \\
\hline $\mathrm{APO}$ & Aspidosperma nobile Müll. Arg. & mo & $\mathrm{cr}$ & $\mathrm{n}$ & 1 & 1 & 1 & 0 & tu & $16^{*}, 20^{*}$ \\
\hline $\mathrm{APO}$ & Aspidosperma polyneuron Müll. Arg. & mo & $\mathrm{cr}$ & $\mathrm{n}$ & 1 & 1 & 1 & 0 & tu & $16^{*}, 20$ \\
\hline $\mathrm{APO}$ & Aspidosperma tomentosum Mart. & mo & $\mathrm{cr}$ & $\mathrm{n}$ & 1 & 1 & 1 & 0 & $\mathrm{tu}$ & 16,20 \\
\hline $\mathrm{APO}$ & Hancornia speciosa Gomez & mo & wh & $\mathrm{n}$ & 0 & 1 & 1 & 0 & tu & $16^{*}, 20$ \\
\hline $\mathrm{APO}$ & Himatanthus obovatus (Müll. Arg.) Woods. & mo & wh & $\mathrm{n}$ & 1 & 1 & 1 & 1 & $\mathrm{tu}$ & 6,20 \\
\hline AST & Eremanthus erythropappus Sch. Bip. & si & wh & $\mathrm{d}$ & 0 & 1 & 1 & 1 & tu & $16^{*}$ \\
\hline AST & Eremanthus glomerulatus Less. & si & wh & $\mathrm{d}$ & 0 & 1 & 1 & 1 & tu & 16 \\
\hline AST & Piptocarpha rotundifolia (Less.) Baker & si & wh & $\mathrm{d}$ & 0 & 1 & 1 & 0 & $\mathrm{tu}$ & 16,17 \\
\hline BIG & Tabebuia aurea (Silva Manso) S. Moore & be & ye & $\mathrm{d}$ & 0 & 1 & 1 & 1 & tu & 4,6 \\
\hline BIG & Tabebuia ochracea (Cham.) Standl. & be & ye & $\mathrm{d}$ & 1 & 1 & 1 & 1 & tu & 4,16 \\
\hline BIG & Zeyheria montana Mart. & $\mathrm{hb}$ & ye & $\mathrm{d}$ & 1 & 0 & 1 & 0 & tu & 1 \\
\hline CAC & Caryocar brasiliense Cambess. & ba & wh & $\mathrm{n}$ & 0 & 1 & 1 & 1 & op & 6,20 \\
\hline CEL & Peritassa campestris (Cambess.) A.C. Sm. & be & $\mathrm{gr}$ & $\mathrm{d}$ & 0 & 1 & 0 & 1 & op & $1^{*}$ \\
\hline CLU & Kielmeyera coriacea Mart. & be & wh & $\mathrm{d}$ & 0 & 1 & 0 & 1 & op & 17,18 \\
\hline CLU & Kielmeyera rubriflora Cambess. & be & wh & $\mathrm{d}$ & 0 & 1 & 0 & 1 & op & 18 \\
\hline $\mathrm{CMB}$ & Buchenavia tomentosa Eichl. & si & $\mathrm{cr}$ & $\mathrm{d}$ & 0 & 0 & 1 & 0 & tu & \\
\hline $\mathrm{CNN}$ & Connarus suberosus Planch. & si & ye & $\mathrm{d}$ & 0 & 1 & 1 & 0 & op & 6,16 \\
\hline $\mathrm{CNN}$ & Rourea induta Planch. & be & wh & $\mathrm{d}$ & 0 & 1 & 1 & 1 & op & 1 \\
\hline DLL & Davilla elliptica A. St-Hil. & be & ye & $\mathrm{d}$ & 0 & 1 & 0 & 1 & op & 1,6 \\
\hline ERX & Erythroxylum campestre A. St-Hil. & si & $\mathrm{cr}$ & $\mathrm{d}$ & 0 & 1 & 1 & 0 & op & 1,3 \\
\hline ERX & Erythroxylum suberosum A. St-Hil. & si & wh & $\mathrm{d}$ & 0 & 0 & 1 & 0 & op & 3,6 \\
\hline ERX & Erythroxylum tortuosum Mart. & si & wh & $\mathrm{d}$ & 0 & 0 & 1 & 0 & op & 3,16 \\
\hline EUP & Manihot caerulescens Pohl & be & $\mathrm{gr}$ & $\mathrm{d}$ & 0 & 0 & 1 & 1 & op & $6^{*}$ \\
\hline FAB & Acosmium subelegans (Mohl.) Yakovlev & be & wh & $\mathrm{d}$ & 1 & 1 & 1 & 0 & op & 6 \\
\hline FAB & Anadenanthera falcata (Benth.) Speg & si & $\mathrm{cr}$ & $\mathrm{d}$ & 0 & 0 & 1 & 1 & op & $17^{*}$ \\
\hline FAB & Andira anthelmia (Vell.) J.F. Macbr. & be & li & $\mathrm{d}$ & 1 & 1 & 1 & 0 & $\mathrm{cl}$ & $6^{*}$ \\
\hline FAB & Andira cuiabensis Benth. & be & li & $\mathrm{d}$ & 1 & 1 & 1 & 0 & $\mathrm{cl}$ & 6 \\
\hline FAB & Andira laurifolia Benth. & be & li & $\mathrm{d}$ & 1 & 1 & 1 & 0 & $\mathrm{cl}$ & $6^{*}$ \\
\hline FAB & Andira vermifuga (Mart.) Benth. & be & li & $\mathrm{d}$ & 1 & 1 & 1 & 0 & $\mathrm{cl}$ & $6^{*}$ \\
\hline FAB & Bauhinia rufa Steud. & ba & wh & $\mathrm{n}$ & 0 & 1 & 1 & 1 & op & 10,17 \\
\hline FAB & Bowdichia virgilioides Kunth & be & li & $\mathrm{d}$ & 1 & 0 & 1 & 0 & $\mathrm{cl}$ & 6,17 \\
\hline FAB & Copaifera langsdorffii Desf. & be & wh & $\mathrm{d}$ & 0 & 1 & 1 & 1 & op & 7,9 \\
\hline FAB & Dimorphandra mollis Benth. & $\mathrm{si}$ & $\mathrm{cr}$ & $\mathrm{d}$ & 0 & 1 & 1 & 0 & op & 6,16 \\
\hline FAB & Dioclea bicolor Benth. & be & li & $\mathrm{d}$ & 1 & 1 & 1 & 1 & op & $8^{*}$ \\
\hline FAB & Hymenaea stigonocarpa Mart. & ba & wh & $\mathrm{n}$ & 1 & 1 & 1 & 1 & op & 6,10 \\
\hline FAB & Machaerium acutifolium Vogel & be & $\mathrm{cr}$ & $\mathrm{d}$ & 1 & 0 & 1 & 0 & $\mathrm{cl}$ & $6^{*}$ \\
\hline
\end{tabular}


TABLE 1

Continued...

\begin{tabular}{|c|c|c|c|c|c|c|c|c|c|c|}
\hline Family & Species & Pol & $\mathrm{CO}$ & $\mathbf{A}$ & RG & $\mathbf{O}$ & $\mathbf{N}$ & $\mathbf{P}$ & $\mathbf{S}$ & Source \\
\hline FAB & Sclerolobium aureum (Tul.) Benth. & si & ye & $\mathrm{d}$ & 0 & 1 & 1 & 0 & op & 6 \\
\hline FAB & Sclerolobium paniculatum Vogel & si & ye & $\mathrm{d}$ & 0 & 1 & 1 & 0 & op & 16,17 \\
\hline FAB & Senna silvestris (Vell.) H.S. Irwin \& Barneby & be & ye & $\mathrm{d}$ & 0 & 1 & 1 & 1 & op & 17,28 \\
\hline FAB & Stryphnodrendon obovatum Benth. & si & wh & $\mathrm{d}$ & 1 & 1 & 1 & 0 & tu & 6,16 \\
\hline FLC & Casearia sylvestris $\mathrm{Sw}$. & si & gr & $\mathrm{d}$ & 0 & 1 & 1 & 1 & op & 1,6 \\
\hline LOG & Strychnos pseudoquina A. St-Hil. & mo & cr & $\mathrm{n}$ & 0 & 1 & 1 & 0 & $\mathrm{tu}$ & 16,20 \\
\hline LYT & Lafoensia pacari A. St-Hil. & ba & ye & $\mathrm{n}$ & 1 & 1 & 1 & 1 & op & 6,23 \\
\hline MLP & Banisteriopsis stellaris (Griseb.) B.Gates & be & wh & $\mathrm{d}$ & 0 & 0 & 0 & 1 & op & $1,24^{*}$ \\
\hline MLP & Byrsonima basiloba A. Juss. & be & ye & $\mathrm{d}$ & 0 & 0 & 0 & 1 & op & $1,24^{*}$ \\
\hline MLP & Byrsonima coccolobifolia A. Juss. & be & li & $\mathrm{d}$ & 0 & 0 & 0 & 1 & op & 2,6 \\
\hline MLP & Byrsonima crassa Nied. & be & ye & $\mathrm{d}$ & 0 & 0 & 0 & 1 & op & 2,17 \\
\hline MLP & Byrsonima intermedia A. Juss. & be & ye & $\mathrm{d}$ & 0 & 0 & 0 & 1 & op & $1,24^{*}$ \\
\hline MLS & Miconia albicans Triana & be & wh & $\mathrm{d}$ & 0 & 0 & 0 & 1 & op & 12,17 \\
\hline MLS & Miconia fallax A. DC. & be & wh & $\mathrm{d}$ & 0 & 1 & 0 & 1 & op & $12,16^{*}$ \\
\hline MLS & Miconia ferruginata A. DC & be & wh & $\mathrm{d}$ & 0 & 1 & 0 & 1 & op & $12^{*}$ \\
\hline MLS & Miconia ligustroides (A. DC.) Naud. & be & wh & $\mathrm{d}$ & 0 & 1 & 0 & 1 & op & 12 \\
\hline MLS & Mouriri elliptica Mart. & be & wh & $\mathrm{d}$ & 0 & 0 & 0 & 1 & op & $11^{*}$ \\
\hline MLV & Eriotheca gracilipes (K. Schum.) A. Robyns & be & wh & $\mathrm{d}$ & 0 & 0 & 1 & 1 & op & 17,19 \\
\hline MLV & Pseudobombax longiflorum (Mart. \& Zucc.) A. Robyns & ba & wh & $\mathrm{n}$ & 0 & 1 & 1 & 1 & op & 6,20 \\
\hline MOR & Brosimum gaudichaudii Trec. & wi & gr & $\mathrm{d}$ & 0 & 0 & 0 & 0 & op & 1,6 \\
\hline MRT & Campomanesia adamantium (Cambess.) O. Berg & be & wh & $\mathrm{d}$ & 1 & 1 & 0 & 1 & op & $1,27^{*}$ \\
\hline MRT & Campomanesia pubescens (A. DC.) O. Berg & be & wh & $\mathrm{d}$ & 1 & 1 & 0 & 1 & op & 1,27 \\
\hline MRT & Eugenia aurata $\mathrm{O}$. Berg & be & wh & $\mathrm{d}$ & 0 & 1 & 0 & 1 & op & $1,6^{*}$ \\
\hline MRT & Eugenia bimarginata A. DC. & be & wh & $\mathrm{d}$ & 0 & 1 & 0 & 1 & op & $1,6^{*}$ \\
\hline MRT & Eugenia piauhiensis O. Berg & be & wh & $\mathrm{d}$ & 0 & 1 & 0 & 1 & op & $1,6^{*}$ \\
\hline MRT & Eugenia punicifolia (Kunth) A. DC. & be & wh & $\mathrm{d}$ & 0 & 1 & 0 & 1 & op & 1 \\
\hline MRT & Myrcia bella Cambess. & be & wh & $\mathrm{d}$ & 0 & 1 & 0 & 1 & op & $6^{*}, 21^{*}$ \\
\hline MRT & Myrcia camapuanensis N.F.E. Silveira & be & wh & $\mathrm{d}$ & 0 & 1 & 0 & 1 & op & $6^{*}, 21^{*}$ \\
\hline MRT & Myrcia crassifolia (O. Berg) Kiaersk. & be & wh & $\mathrm{d}$ & 0 & 1 & 0 & 1 & op & $6^{*}, 21^{*}$ \\
\hline MRT & Myrcia fallax (Rich.) A. DC. & be & wh & $\mathrm{d}$ & 0 & 1 & 0 & 1 & op & $6^{*}, 21^{*}$ \\
\hline MRT & Myrcia guianensis A. DC. & be & wh & $\mathrm{d}$ & 0 & 1 & 0 & 1 & op & $6^{*}, 21^{*}$ \\
\hline MRT & Myrcia laruotteana Cambess. & be & wh & $\mathrm{d}$ & 0 & 1 & 0 & 1 & op & $6^{*}, 21^{*}$ \\
\hline MRT & Myrcia linguaeformis Kiaersk. & be & wh & $\mathrm{d}$ & 0 & 1 & 0 & 1 & op & $6^{*}, 21^{*}$ \\
\hline MRT & Myrcia rhodeosepala Kiaersk. & be & wh & $\mathrm{d}$ & 0 & 1 & 0 & 1 & op & 21 \\
\hline MRT & Myrcia sp. nov. & be & wh & $\mathrm{d}$ & 0 & 1 & 0 & 1 & op & $6^{*}, 21^{*}$ \\
\hline MRT & Myrcia torta A. DC. & be & wh & $\mathrm{d}$ & 0 & 1 & 0 & 1 & op & $6^{*}, 21^{*}$ \\
\hline MRT & Myrcia uberavensis $\mathrm{O}$. Berg & be & wh & $\mathrm{d}$ & 0 & 1 & 0 & 1 & op & 1 \\
\hline MRT & Psidium laruotteanum Cambess. & be & wh & $\mathrm{d}$ & 0 & 1 & 0 & 1 & op & $6^{*}, 21^{*}$ \\
\hline NYC & Guapira noxia (Netto) Lundell & si & wh & $\mathrm{d}$ & 0 & 1 & 1 & 0 & $\mathrm{tu}$ & 16,17 \\
\hline $\mathrm{NYC}$ & Neea theifera Oerst. & si & ye & $\mathrm{d}$ & 0 & 0 & 1 & 0 & tu & 6,16 \\
\hline $\mathrm{OCH}$ & Ouratea acuminata (A. DC.) Engl. & be & ye & $\mathrm{d}$ & 0 & 1 & 0 & 1 & op & $1,6^{*}$ \\
\hline $\mathrm{OCH}$ & Ouratea castaneaefolia (A. DC.) Engl. & be & ye & $\mathrm{d}$ & 0 & 1 & 0 & 1 & op & 6,17 \\
\hline $\mathrm{OCH}$ & Ouratea semiserrata (Mart. \& Nees) Engl. & be & ye & $\mathrm{d}$ & 0 & 1 & 0 & 1 & op & 6 \\
\hline $\mathrm{OCH}$ & Ouratea spectabilis (Mart.) Engl. & be & ye & $\mathrm{d}$ & 0 & 1 & 0 & 1 & op & $1,6^{*}$ \\
\hline PRT & Roupala montana Aubl. & mo & wh & $\mathrm{n}$ & 0 & 1 & 1 & 0 & op & 6,20 \\
\hline RUB & Chomelia ribesioides Benth.ex A. Gray & mo & wh & $\mathrm{d}$ & 0 & 1 & 1 & 0 & $\mathrm{tu}$ & $6^{*}$ \\
\hline RUB & Palicourea rigida Kunth & $\mathrm{hb}$ & or & $\mathrm{d}$ & 0 & 0 & 1 & 0 & tu & 1,16 \\
\hline RUB & Tocoyena formosa (Cham. \& Schltdl.) K. Schum. & mo & ye & $\mathrm{n}$ & 0 & 1 & 1 & 0 & tu & 6,16 \\
\hline SPT & Pouteria ramiflora (Mart.) Radlk. & mo & $\mathrm{cr}$ & $\mathrm{n}$ & 0 & 1 & 0 & 1 & tu & 25 \\
\hline SPT & Pouteria torta (Mart.) Radlk. & mo & $\mathrm{cr}$ & $\mathrm{n}$ & 0 & 1 & 0 & 1 & tu & 25 \\
\hline STY & Styrax ferrugineum Nees \& Mart. & be & wh & $\mathrm{d}$ & 0 & 1 & 1 & 0 & tu & 16,22 \\
\hline VOC & Qualea grandiflora Mart. & mo & ye & $\mathrm{n}$ & 0 & 1 & 1 & 1 & tu & 6,20 \\
\hline $\mathrm{VOC}$ & Qualea multiflora Mart. & be & wh & $\mathrm{d}$ & 1 & 1 & 1 & 0 & op & 16,17 \\
\hline VOC & Qualea parviflora Mart. & be & li & $\mathrm{d}$ & 1 & 0 & 1 & 0 & op & 6,16 \\
\hline $\mathrm{VOC}$ & Vochysia thyrsoidea Pohl & be & ye & $\mathrm{d}$ & 1 & 0 & 1 & 1 & op & 15,16 \\
\hline
\end{tabular}




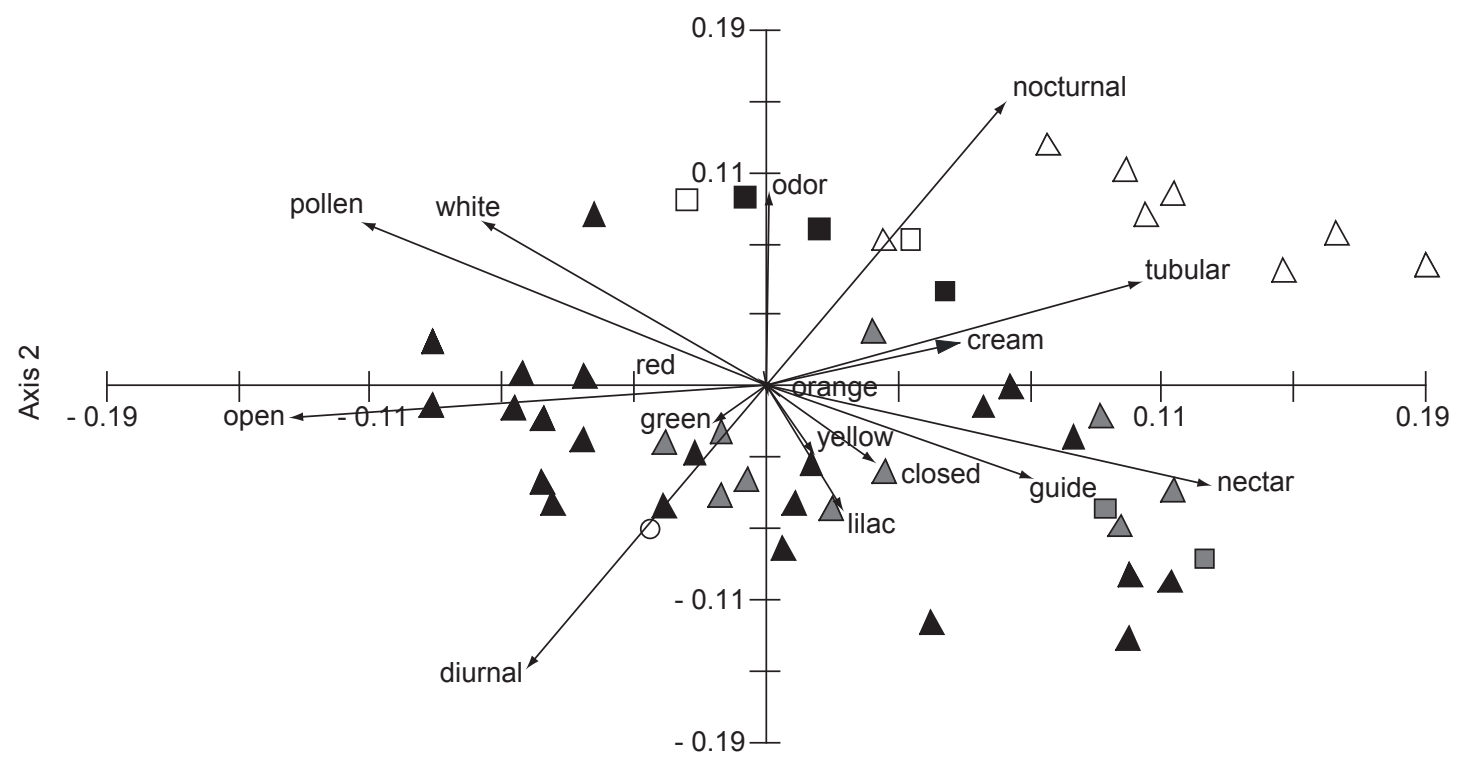

Axis 1

Fig. 1 - Principal component analysis of floral traits and woody species of the cerrado fragments in Upper Taquari region (central-western Brazil). Species were classified according to their pollination system: $\boldsymbol{\Delta}=$ bees; $\boldsymbol{\Delta}=$ small insects; $\triangle$ = moths; $\mathbf{\square}=$ bats; $\mathbf{\square}=$ hummingbirds; $\square$ = beetles; and $\bigcirc=$ wind.

Gottsberger, 1988; Bawa, 1990; Barbosa, 1997; Oliveira \& Gibbs, 2000). Flowers pollinated mainly by beetles were yellow, closed, nocturnal, and with odor; flowers pollinated mainly by birds were orange, tubular, diurnal, and with nectar; flowers pollinated mainly by bats were white, nocturnal, and with odor; and flowers pollinated mainly by moths were pale, tubular, nocturnal, and with odor and nectar. Most of the flowers pollinated mainly by bees were open and yellow, white, cream, or lilac. Such colors, as well as the sweet and pleasant odor emitted by the flowers during the anthesis, are attributes related to the attraction of bees, since they are sensitive to olfactory and visual stimuli (Faegri \& Pijl, 1979; Kevan \& Baker, 1983).

Flowers pollinated mainly by small insects were small, usually open, and green or yellow. On the one hand, these traits imply floral rewards that are accessible to many insects; on the other hand, a certain insect from such a group can visit flowers of several species, since there is a lot of species with these traits (Bawa \& Opler, 1975; Bawa, 1980). Flowers pollinated by this group are generally small (less than $1 \mathrm{~cm}$ in length), pale green or pale yellow, and lack morphological specialization with the result that floral rewards are accessible to a wide variety of insects (Bawa et al., 1985). We found a large overlapping between the two most frequent groups, bees and small insects, which probably occurred due to the absence of specificity, which is the most frequent condition in different vegetation types (Bawa, 1980), including the cerrado (Borges, 2000). In tropical forests, many species have morphologically simple flowers, allowing the access of different categories of visitors, such as bees, butterflies, moths, flies, and wasps (Bawa, 1990). Even species with more complex flowers are visited by several species that act as pollinators (Bawa, 1990).

In the cerrado, bird- and wind-pollinated flowers are rare (Silberbauer-Gottsberger \& Gottsberger, 1988; Oliveira, 1991) and were the groups with lowest frequencies in the area we studied as well. Although hummingbirds are important pollinators of herbs in open cerrado areas (Silberbauer-Gottsberger \& Gottsberger, 1988; Barbosa, 1997), they seem to be only secondary or opportunistic visitors of cerrado woody 
species (Oliveira \& Gibbs, 2000). The only species pollinated by wind that we sampled had inconspicuous flowers, without odor and rewards, corroborating Faegri \& Pijl (1979), who related these traits to abiotic pollination. Wind pollination, which has been associated with seasonally dry areas (Bullock, 1994), is rare among cerrado woody species and occurs commonly only among grasses and sedges (Silberbauer-Gottsberger \& Gottsberger, 1988; Oliveira \& Gibbs, 2002). We did not find butterfly-pollinated flowers, which are poorly represented among cerrado woody species (Oliveira \& Gibbs, 2000; 2002).

Information from studies on pollination in cerrado sites (Silberbauer-Gottsberger \& Gottsberger, 1988; Oliveira, 1991; Oliveira \& Gibbs, 2000; 2002) pointed out the existence of several pollination systems, suggesting that plant species depend on the interaction with several groups of animals, and vice-versa. Our ordination analysis of floral traits and plant species showed that there was a grouping among species with some pollination systems (beetles, moths, birds, and bats), for which inferences based on the floral characters are recommended for cerrado sites. In these cases, one could infer the pollination system based on the floral traits. On the contrary, regarding the species pollinated mainly by bees or small insects, inferences based on the floral traits are not recommended, due to the large dispersion of the species scores and overlapping between these two classes, which occurred, probably, due to the specificity absence in the plant-pollinator relationships.

Acknowledgments - We are grateful to $\mathrm{CNPq}$ and Conservation International, especially Drs. Mário Barroso Ramos-Neto and Paulo Gustavo do Prado Pereira, for financial support; to the Emas and Oréades Foundations, for satellite images; to André Vitor Fleuri Jardim and Júlia Caram, for help in the field; and to an anonymous referee, for useful suggestions.

\section{REFERENCES}

BARBOSA, A. A., 1997, Biologia reprodutiva de uma comunidade de campo sujo, Uberlândia - MG., Ph.D. Thesis - Universidade Estadual de Campinas, Brasil.

BARROS, M. G., 1992, Fenologia da floração, estratégias reprodutivas e polinização de espécies simpátricas do gênero Byrsonima Rich (Malpighiaceae). Rev. Brasil. Biol., 52: 343-353.

BARROS, M. G., 1998, Sistemas reprodutivos e polinização em espécies simpátricas de Erythroxylum
P. Br. (Erythroxylaceae) do Brasil. Rev. Brasil. Bot., 21: $159-166$.

BARROS, M. G., 2001, Pollination ecology of Tabebuia aurea (Manso) Benth. \& Hook. and T. ochracea (Cham.) Standl. (Bignoniaceae) in Central Brazil cerrado vegetation. Rev. Brasil. Bot., 24: 255-261.

BATAlHA, M. A. \& MANTOVANI, W., 1999, Chaves de identificação das espécies vegetais vasculares baseada em caracteres vegetativos para a ARIE Cerrado Pé-deGigante (Santa Rita do Passa Quatro, SP). Rev. Inst. Flor, 11: $137-158$.

BATALHA, M. A. \& MARTINS, F. R., 2002, The vascular flora of the cerrado in Emas National Park (Goiás, central Brazil). Sida, 20: 295-312.

BAWA, K. S., 1980, Evolution of dioecy in flowering plants. Ann. Rev. Ecol. Syst., 11: 15-39.

BAWA, K. S., 1990, Plant-pollinator interactions in tropical rain forests. Ann. Rev. Ecol. Syst., 21: 399-422.

BAWA, K. S., BULLOCH, S. H., PERRY, D. R., COVILLE, R. E. \& GRAYUM, M. H., 1985, Reproduction biology of tropical lowland rain forest tree. II. Pollination system. Am. J. Bot., 72: 346-356.

BAWA, K. S. \& OPLER, P. A., 1975, Dioecism in tropical forest trees. Evolution, 29: 167-179.

BORGES, H. B. N., 2000, Biologia reprodutiva e conservação do estrato lenhoso numa comunidade do cerrado. Ph.D. Thesis - Universidade Estadual de Campinas, Brasil.

BOSCH, J., RETANA, J. \& CERDÁ, X., 1997, Flowering phenology, floral traits and pollinator composition in a herbaceous Mediterranean plant community. Oecologia, 109: 583-591.

BOURLIÈRE, F. \& HADLEY, M., 1983, Present-day savannas: an overview, pp. 1-17. In: D. W. Goodall (ed.), Ecosystems of the world - tropical savannas, Amsterdam, Elsevier.

BULLOCK, S. H., 1994, Wind pollination of Neotropical dioecious trees. Biotropica, 26: 172-179.

COUTINHO, L. M., 1990, Fire in the ecology of the Brazilian cerrado, pp. 82-105. In: J. G. Goldammer (ed.), Fire in the tropical biota, Springer, Berlin.

CRESTANA, C. S. M. \& KAGEYAMA, P. Y., 1989, Biologia reprodutiva de Copaifera langsdorffii. Rev. Inst. Flor., 1: 201-214.

ENDRESS, P. K., 1994, Diversity and evolutionary biology of tropical flowers. Cambridge University, Cambridge.

FAEGRI, K. \& PIJL, L. van der, 1979, The principles of pollination ecology. Pergamon, New York.

FRANCO, A. L. M., 1995, Ecologia da polinização e biologia reprodutiva de sete espécies de Phaseoleae. Ph.D. Thesis Universidade Estadual de Campinas, Brasil.

FREITAS, C. V. \& OLIVEIRA, P. E., 2002, Biologia reprodutiva de Copaifera langsdorffi Desf. (Leguminosae, Caesalpinioideae). Rev. Brasil. Bot., 25: 311-321.

GIBBS, P. E., OLIVEIRA, P. E. \& BIANCHI, M. B., 1999, Postzygotic control of selfing in Hymenaea stigonocarpa (Leguminosae-Caesalpinioideae), a bat-pollinated tree of the Brazilian cerrados. Int. J. Plant Sci., 160: 72-78. 
GOLDENBERG, R., 1994, Estudos sobre a biologia reprodutiva de espécies de Melastomataceae de Cerrado em Itirapina, $S P$. M.Sc. Dissertation - Universidade Estadual de Campinas, Brasil.

GOTTSBERGER, G., 1994, As anonáceas do cerrado e a sua polinização. Rev. Brasil. Biol., 54: 391-402.

GOLDENBERG, R. \& SHEPHERD, G. J., 1998, Studies on the reproductive biology of Melastomataceae in "cerrado" vegetation. Plant Syst. Evol., 211: 13-29.

GOTTSBERGER, G., 1999. Pollination and evolution in neotropical Annonaceae. Pl. Sp. Biol., 14: 143-152.

HERRERA, C. M., 1996, Floral traits and plant adaptation to insect pollinators: a devil's advocate approach, pp. 65-87. In: D. G. Lloyd \& S. C. H. Barrett (eds.), Floral biology: studies on floral evolution in animal pollinated plants, Chapman \& Hall, New York.

JONGMAN, R. H. G., TER BRAAK, C. J. F. \& VAN TONGEREN, O. F. R., 1995, Data analysis in community and landscape ecology. Cambridge University, Cambridge.

JUDD, W. S., CAMPBELL, C. S., KELlOG, E. A. \& STEVENS, P. F., 1999, Plant systematics: a phylogenetic approach. Sinauer, Sunderland.

KEVAN, P. G. \& BAKER, H. G., 1983, Insects as flowers visitors and pollinators. Annu. Rev. Entomol., 28: 407-453.

KÖPPEN, W., 1948, Climatología. Fondo de Cultura Económica, México.

KOVACH, 1999, Multivariate Statistical Package v. 3.1. Kovach Computing Services, Anglesey.

KRESS, W. J. \& BEACH, J. H., 1994, Flowering plant reproductive systems, pp. 161-182. In: L. A. McDade, K. S. Bawa, H. A. Hespenheide \& G. S. Hartshorn (eds.), La Selva. Ecology and natural history of neotropical rain forest, University Chicago, Chicago.

LEVIN, D. A. \& ANDERSON, W. W., 1970, Competition for pollinators between simultaneously flowering species. Am. Nat., 104: 455-467.

MACHADO, A. O. \& OLIVEIRA, P. E., 2000, Biologia floral e reprodutiva de Casearia grandiflora Camb. (Flacourtiaceae). Rev. Brasil. Bot., 23: 283-290.

MOLDENKE, A. R. \& LINCOLN, P. G., 1979, Pollination ecology in montane Colorado. Phytologia, 42: 349-379.

MUELLER-DOMBOIS, D. \& ELLENBERG, H., 1974, Aims and methods of vegetation ecology. John Willey \& Sons, New York.

OLIVEIRA, P. E., 1991, The pollination and reproductive biology of a cerrado woody community in Brazil. Ph.D. Thesis - University of St. Andrews, St. Andrews.

OLIVEIRA, P. E. \& GIBBS, P. E., 1994, Pollination biology and breeding systems of six Vochysia species (Vochysiaceae) in Central Brazil. J. Trop. Ecol., 10: 509-522.

OLIVEIRA, P. E. \& GIBBS, P. E., 2000, Reproductive biology of woody plants in a cerrado community of Central Brazil. Flora, 195: 311-329.

OLIVEIRA, P. E. \& GIBBS, P. E., 2002, Pollination and reproductive biology in cerrado plant communities, pp. 329-347. In: P. E. Oliveira \& R. J. Marquis (eds.),
The cerrados of Brazil: Ecology and Natural History of a Neotropical Savanna, Columbia University, New York.

OLIVEIRA, P. E., GIBBS, P. E. \& BARBOSA, A. A., 2004, Moth pollination of woody species in the Cerrados of Central Brazil: a case of so much owed to so few? Plant Syst. Evol., 245: 41-54.

OLIVEIRA, P. E., GIBBS, P. E., BARBOSA, A. A. \& TALAVERA, S., 1992, Contrasting breeding systems in two Eriotheca (Bombacaceae) species of the Brazilian cerrados. Plant Syst. Evol., 179: 207-219.

OLIVEIRA, P. E. \& PAULA F. R., 2001, Fenologia e biologia reprodutiva de plantas de Matas de galeria, pp. 303-331. In: J. F. Ribeiro, C. E. L. Fonseca da \& J. C. Souza-Silva (eds.), Cerrado: caracterização e recuperação de Matas de Galeria, Embrapa, Planaltina.

OLIVEIRA, P. E. \& SAZIMA, M., 1990, Pollination biology of two species of Kielmeyera (Guttiferae) from Brazilian cerrado vegetation. Plant Syst. Evol., 172: 35-49.

PROCTOR, M., YEO, P. \& LACK, A., 1996, The natural history of pollination. Harper Collins, New York.

PROENÇA, C. E. B. \& GIBBS, P. E., 1994, Reproductive biology of eight sympatric Myrtaceae from Central Brazil. New Phytol., 126: 343-354.

RAMIREZ, N. \& BRITO, Y., 1992, Pollination biology in a palm swamp community in the Venezolana central plains. Bot. J. Linn. Soc., 110: 277-302.

RAMOS-NETO, M. B. \& PIVELLO, V. R., 2000, Lightning fires in a Brazilian savanna National Park: rethinking management strategies. Environ. Manage., 26: 675-684.

RATTER, J. A., RIBEIRO, J. F. \& BRIDGEWATER, S., 1997, The Brazilian cerrado vegetation and threats to its biodiversity. Ann. Bot., 80: 223-230.

SARAIVA, L. C., CESAR, O. \& MONTEIRO, R., 1988, Biologia da polinização e sistemas de reprodução de Styrax camporum Pohl e S. ferrugineus Nees et Mart. (Styracaceae). Rev. Brasil. Bot., 11: 71-80.

SAZIMA, M. \& SAZIMA, I., 1975, Quiropterofilia em Lafoensia pacari St. Hil. (Lythraceae), na Serra do Cipó, Minas Gerais. Ciênc. Cult., 27: 405-416.

SCHOLES, R. J. \&ARCHER, S. R., 1997, Tree-grass interactions in savannas. Annu. Rev. Ecol. Syst., 28: 517-544.

SIGRIST, M. R., 2001, Biologia reprodutiva de doze espécies simpátricas de Malpighiaceae em mata semidecídua do sudoeste Brazileiro. Ph.D. Thesis - Universidade Estadual de Campinas, Brasil.

SILBERBAUER-GOTTSBERGER, I. \& GOTTSBERGER, G., 1988, A polinização de plantas do cerrado. Rev. Brasil. Biol., 48: 651-663.

SILBERBAUER-GOTTSBERGER, I., GOTTSBERGER, G. \& WEBBER, A. C., 2003, Morphological and functional flower characteristics of New and Old World Annonaceae with respect to their mode of pollination. Taxon, 52: 701-718.

SMA. Secretaria de Estado do Meio Ambiente, 1997, Cerrado: bases para conservação e uso sustentável das áreas de cerrado do estado de São Paulo. Secretaria de Estado do Meio Ambiente, São Paulo. 
TOREZAN-SILINGARDI, H. M. \& DEL-CLARO, K., 1998, Behavior of visitors and reproductive biology of Campomanesia pubescens (Myrtaceae) in cerrado vegetation. Ciênc. Cult., 50: 281-284.

VIANA, B. F., KLEINERT, A. M. P. \& IMPERATRIZFONSECA, V. L., 1997, Abundance and flower visits of bees in a Cerrado of Bahia, Tropical Brazil. Stud. Neotrop. Fauna Environ., 32: 212-219.

WANDERLEY, M. G. L., SHEPHERD, G. J., GIULIETTI, A. M. \& MELHEM, T. S., 2002, Flora Fanerogâmica do Estado de São Paulo. $2^{\circ}$ vol. Fapesp e Hucitec, São Paulo.

WANDERLEY, M. G. L., SHEPHERD, G. J. \& GIULIETTI, A. M., 2003, Flora Fanerogâmica do Estado de São Paulo. $3^{\circ}$ vol. Fapesp e Hucitec, São Carlos.

WASER, N. M., 1983, The adaptive nature of floral traits: ideas and evidence, pp. 241-285. In: L. Real (ed.), Pollination biology, Academic, New York.
WASER, N. M., CHITTKA, L., PRICE, M. V., WILLIAMS, N. M. \& OLLERTON J., 1996, Generalization in pollination systems, and why it matters. Ecology, 77: 1043-1060.

WEBER, W. A., 1982, Mnemonic three-letter acronyms for the families of vascular plants: a device for more effective herbarium curation. Taxon, 31: 74-88.

WILSON, P., CASTEllanos, M. C., HOGUE, J. N., THOMSON, J. D. \& ARMBRUSTER, W. S., 2004, A multivariate search for pollination syndromes among penstemons. Oikos, 104: 345-361.

WUNDERLEE, J. M., 1997, The role of animal seed dispersal in accelerating native forest regeneration on degraded tropical lands. Forest Ecol. Manag., 99: 223-235. 